\title{
MIGRACIÓN ENTRE OCHO REGIONES DE MÉXICO, 1955-1970
}

\author{
Virgilio Partida Bush* \\ El Colegio de México
}

\section{INTRODUCCIÓN}

LA MAYOR PARTE DE LOS ESTUDios que se han hecho en México para cuantificar la migración interna, consisten en estimaciones residuales de saldos netos migratorios. ${ }^{1}$ Apenas dos (Tabah y Cosío, 1970; Partida, 1982) contemplan el fenómeno desde una óptica interregional que cubre al país en su totalidad.

$\mathrm{La}$ escasez de estimaciones de intercambios de población entre regiones se debe en gran parte a la poca información disponible. Los tres últimos censos de población $(1960,1970$ y 1980) constituyen la

\footnotetext{
*El autor agradece la colaboración de Juan Manuel Rico López en el procesamiento electrónico de la información.

1 Cabrera (1967, 1975 y 1981), entre otros, ha obtenido saldos netos migratorios para las 32 entidades federativas durante la década de los cincuenta, mediante diferentes métodos; para una regionalización propia con base en las zonas de salarios mínimos para las décadas de los cincuenta y los sesenta, y para todas las entidades federativas y hs principales ciudades del país en cada una de las tres décadas del periodo 1930-1960, respectivamente. Y, Ordorica et al. (1976) los ha obtenido para todas las entidades federativas para la década de los sesenta, mediante diferentes metodos.
} 
única fuente de datos sobre flujos migratorios interregionales para todo el país, por haberse incluido en ellos preguntas específicas para dicho fin; pero sólo para el recuento de 1970 se han publicado las tabulaciones correspondientes. Aunque ahí, la falta de un cuadro que dé cuenta de migrantes recientes por edad, parece haber sido la principal limitante para obtener flujos migratorios interregionales, reduciendo entonces las cuantificaciones a estimaciones residuales de la migración neta.

La migración no es un fenómeno estable, cambia con el tiempo de acuerdo a como cambia la estructura socioeconómica, aunque determinadas corrientes se mantienen como las más cuantiosas y las más importantes. Es por esto que, en este artículo, se presentan estimaciones de flujos migratorios y tasas del fenómeno para los tres quinquenios comprendidos entre 1955 y $1970 .^{2}$

Ya no sólo es el éxodo rural el flujo migratorio más importante a considerar; son los movimientos poblacionales entre regiones los que permiten planear e incluso modificar, mediante políticas específicas, la distribución de la población sobre el territorio mexicano. No se trabajaron aquí flujos interestatales, debido al escaso e incluso nulo tamaño de varias corrientes migratorias de este tipo. Sólo se obtuvieron estimaciones de la migración en un sistema de ocho regiones -el más usado en estudios demográficos para México- que gráficamente se presenta en el mapa 1.

La estimación parte de la idea original del modelo multirregional de Rogers (1968; 1975), y se presenta únicamente a nivel global, es decir, sin distinguir edad ni sexo. ${ }^{3}$ En el segundo apartado se presenta el modelo y su aplicación para las ocho regiones de México y para los tres quinquenios considerados. $Y$ en el tercero, un análisis de la evolución en el tiempo del volumen y distribución espacial de los flujos interregionales, de sus tasas y de su contribución al crecimiento demográfico total, de cada una de las regiones.

2 No se hicieron las estimaciones correspondientes a los dos quinquenios de la década de los setenta, debido a que aún no se dispone de los resultados definitivos del censo de la población de 1980 para el Distrito Federal y para el estado de México.

3 Dentro del proyecto Análisis multüregional de la población de México. Tendencias y perspectivas, a cargo del autor, en el Centro de Estudios Demográficos y de Desarrollo Urbano de El Colegio de México, se ha comenzado a hacer la estimación de flujos migratorios por edad y sexo, interregionales e interestatales, para el periodo 1960-1980. 


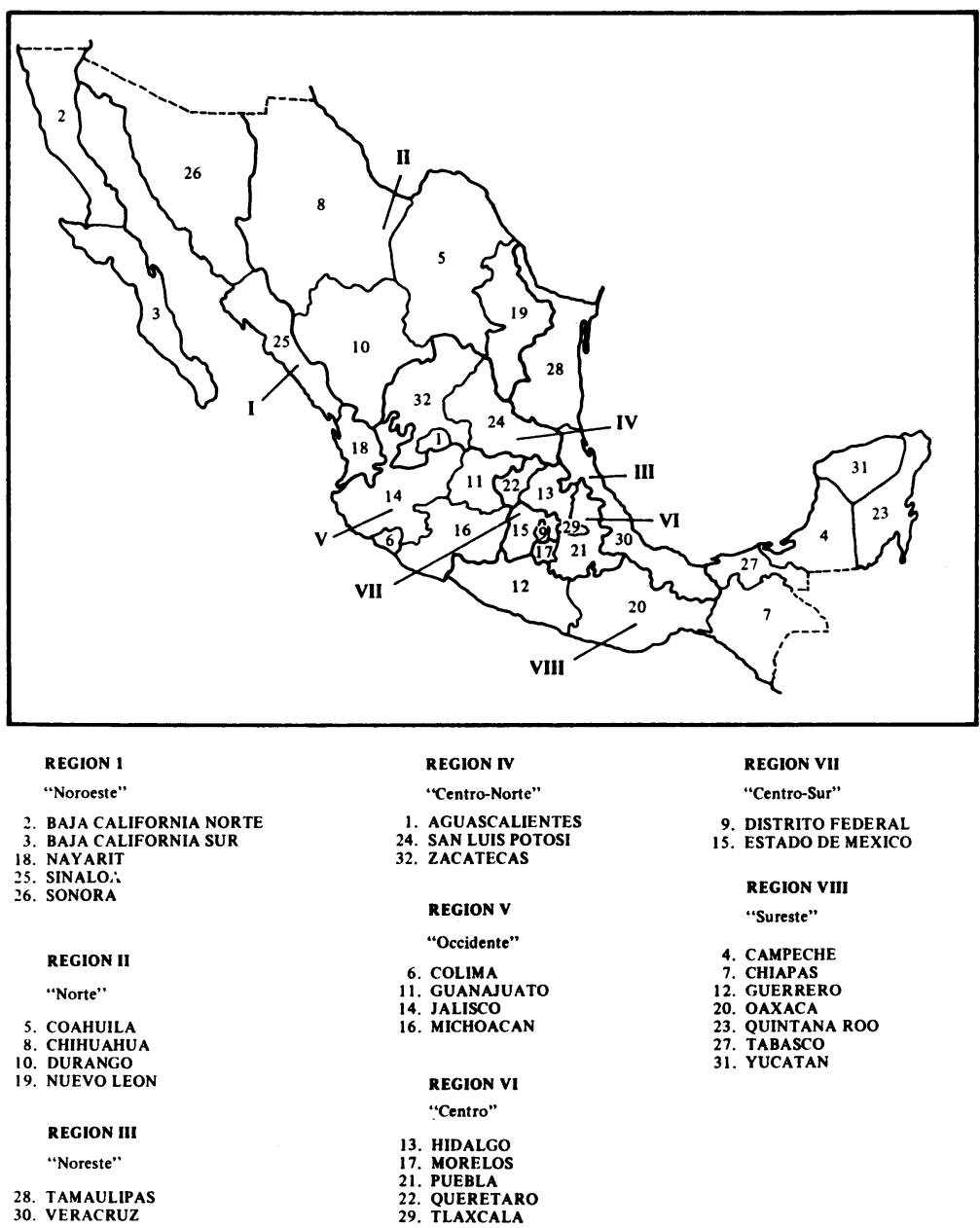




\section{El modelo y SU APLicación a OCHO REgIONES DE MÉXICO}

Sea $N_{i}(t)$ la población total residente en la región $i$ al tiempo que $t$ y $N_{i}(t+5)$ es la residente cinco años después. En el caso de México que aquí se trabaja, $i$ toma valores de 1 a 8 (mapa 1) y $t$ de 1955 , 1960 y 1965 . Sean $K_{i j}$ los emigrantes desde la región $i$ hacia la región $j$ durante el quinquenio $(t, t+5)$. Entonces, dado que se consideran ocho regiones, el total de inmigrantes hacia $i$ durante el quinquenio será:

$$
K_{. i}=\sum_{j \neq i}^{8} K_{j i}
$$

$y$, el total de emigrantes desde $i$ :

$$
K_{i .}=\underset{j \neq i}{\sum} K_{i j}
$$

Si $B_{i}$ y $D_{i}$ denotan los nacimientos y las defunciones ocurridos en la región $i$, respectivamente durante el quinquenio $(t, t+5)$, por la ecuación compensadora se tiene:

$$
\begin{array}{r}
N_{i}(t+5)=N_{i}(t)+B_{i}-D i+K_{. i}-K_{i .} \\
=N_{i}(t)+B_{i}-D_{i}+\underset{j \neq i}{\sum_{j i}^{8} K_{j i}-\sum_{j \neq i}^{8} K_{i j}}
\end{array}
$$

y si se considera el crecimiento natural o vegetativo en la región $i$ en su conjunto en lugar de sus componentes, es decir. $C N_{i}=B_{i}-D_{i}$, la ecuación anterior se convierte en:

$$
N_{i}(t+5)-N_{i}(t)=C N_{i}+\sum_{j \neq i}^{8} K_{j i} \underset{j \neq i}{\sum_{j \neq i}^{8}} K_{i j}
$$

Sea $L_{j}$ el número de años-persona vividos por la población en la región $i$ durante el quinquenio $(t, t+5)$, es decir, 


$$
L_{i}=\int_{t}^{t+5} N(y) \cdot d y
$$

entonces, por la definición de tasa -cociente que resulta de dividir los eventos ocurridos en un intervalo de tiempo entre los años-persona vividos por la población durante el mismo intervalo-, la bruta de crecimiento natural en la región $i$ es $c n_{i}=C N_{i} / L_{i}$, y de emigración desde la región $i$ hacia la región $j$ es $M_{i j}=K_{i j} / L_{i}$. Sustituyendo estas tasas en (1) se tiene:

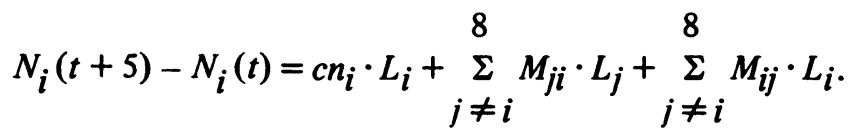

Sea $\bar{N}(t)$ un vector columna de orden 8 cuyo $i$-ésimo elemento es $N_{i}(t), \bar{N}(t+5)$ un vector columna de orden 8 cuyo $i$-ésimo elemento es $N_{i}(t+5)$, y $\bar{L}$ otro también de orden 8 cuyo $i$-ésimo elemento es $L_{i}$, es decir, el tiempo vivido en la región $i$ entre $t$ y $t+5$, o sea,

$$
\bar{N}(t)=\left[\begin{array}{c}
N_{1}(t) \\
N_{2}(t) \\
\cdot \\
\cdot \\
N_{8}(t)
\end{array}\right], \quad \bar{N}(t+5)=\left[\begin{array}{c}
N_{1}(t+5) \\
N_{2}(t+5) \\
\cdot \\
\cdot \\
\cdot \\
N_{8}(t+5)
\end{array}\right] \text { y } \bar{L}=\left[\begin{array}{c}
L_{1} \\
L_{2} \\
\cdot \\
\cdot \\
\cdot \\
L_{8}
\end{array}\right]
$$

y, sea $M$ una matriz cuadrada de orden 8 definida como:

$$
\sim=\left[\begin{array}{cccc}
-c n_{1}+\sum_{j \neq 1}^{8} M_{1 j} & -M_{21} & \ldots & -M_{81} \\
-M_{12} & -c n_{2}+\sum_{j \neq 2}^{8} M_{2 j} \ldots & -M_{82} \\
\cdot & \cdot & \cdot & \cdot \\
\cdot & \cdot & \cdot & \cdot \\
-M_{18} & -M_{28} & \ldots-c n_{8}+\sum_{J \neq}^{8} M_{8 j}
\end{array}\right]
$$


es claro entonces que, la ecuación matricial

$$
\bar{N}(t+5)-\bar{N}(t)=-\underset{\sim}{\sim} \cdot \bar{L}
$$

satisface la ecuación (3) para cualquier región $i$.

En la ecuación anterior se tiene un término, el vector $\bar{L}$ que contiene los años-persona vividos por la población en cada una de las ocho regiones, valores diffíciles de obtener en la realidad. Para estimar estos tiempos vividos, se hace un supuesto muy común en demografía: los eventos (nacimientos, defunciones y migraciones) se distribuyen uniformemente durante el quinquenio, con lo cual, por (2):

$$
\bar{L}=\int_{t}^{t+5} \bar{N}(y) \cdot d y \doteq \frac{5}{2}\{\bar{N}(t)+\bar{N}(t+5)\},
$$

que sustituyéndolo en (5) da:

$$
\bar{N}(t+5)-N(t)=-\frac{5}{2} \cdot \underset{\sim}{M}\{\bar{N}(t)+\bar{N}(t+5)\},
$$

Sea $\underset{\sim}{G}$ una matriz cuadrada de orden 8 tal que satisface:

$$
\bar{N}(t+5)=G \cdot \bar{N}(t)
$$

donde, $g_{i j}$, elemento en la $i$-ésima columna y en el $j$-ésimo renglón, denota la proporción por la que, cada persona residente en la región $i$ al inicio del quinquenio (al tiempo $t$ ), incrementa la población en la región $j$ al final del quinquenio (al tiempo $t+5$ ). A esta matriz $G$, Rogers (1968:7) la denomina "operador de crecimiento y distribución espacial de la población".

Sustituyendo (7) en (6) se tiene:

$$
\underset{\sim}{G} \cdot \bar{N}(t)-\bar{N}(t)=-\frac{5}{2} \cdot \underset{\sim}{M}\{\bar{N}(t)+\underset{\sim}{G} \cdot \bar{N}(t)\},
$$

de donde,

$$
\left.\{\underset{\sim}{I}-\underset{\sim}{\sim}\} \bar{N}(t)=\frac{5}{2} \cdot \underset{\sim}{M} \underset{\sim}{I}+\underset{\sim}{\sim}\right\} \bar{N}(t)
$$

o bien

$$
\{\underset{\sim}{I}-G\}=\frac{5}{2} \cdot M\{\underset{\sim}{I}+\underset{\sim}{\sim}\}
$$


$y$, finalmente

$$
M=\frac{2}{5}\{\underset{\sim}{I}-G\}\{\underset{\sim}{I}+\underset{\sim}{G}\}^{-1}
$$

donde, $\underset{\sim}{I}$ es la matriz identidad de orden 8 y -1 denota la inversa.

Para obtener las tasas de migración contenidas en la matriz $M$ es necesario conocer el operador $G$, caso inverso al más común, donde el segundo se deduce a partir de la primera. Una vez determinadas las tasas, tanto de crecimiento natural como de emigración, se multiplican por el tiempo vivido y se obtienen el crecimiento natural absoluto y los flujos migratorios interregionales para cada quinquenio.

¿Cómo encontrar el operador $G$ ? De la definición de $g_{i j}$, el producto $g_{i j} \cdot N_{i}(t)$ representa a las personas que al residir en la región $i$ al inicio del quinquenio -o nacer durante el quinquenio (en $i$ o en j) de progenitores que se encontraban al inicio del lustro en la región $i$ - sobreviven en la región $j$ al final del quinquenio. Si a estos "flujos migratorios" los denotamos por $G_{i j}$, es claro entonces que $g_{i j}=G_{i j} /$ $N_{i}(t)$.

Para determinar esas $G_{i j}$ se necesita información que indique las personas que al haber residido en $i$ al tiempo $t$-o bien nacido en $i$ durante el quinquenio o en $j$ de padres que se encontraban al inicio de él en $i$ - residan en $j$ al tiempo $t+5$, y estos datos no son disponibles para México.

En los censos de población de 1960 y 1970 se preguntó, por un lado, por el tiempo que cada persona tenía residiendo en la entidad federativa en que fue entrevistada, y, por otro, la entidad federativa de su residencia anterior.

Suponiendo que los $G_{i j}$ para los quinquenios $1955-1960$ y 1965 1970 se pudieran aproximar a estos migrantes, captados para el quinquenio previo por los censos de 1960 y 1970 respectivamente, se podrían entonces obtener los operadores matriciales $\underset{\sim}{G}$ para esos dos lustros. Este supuesto equivale a suponer que, durante el quinquenio previo al censo, hubo una sola migración o ninguna. Si bien esto parece no ser cierto en la realidad, dado lo relativamente amplio de un intervalo de tiempo como cinco años, la falta de información más precisa mencionada, no nos deja otra alternativa. Sin embargo, un periodo de cinco años puede parecer también lo relativamente corto, como para que las migraciones múltiples sean tan significativas que originen la asignación errónea de la región de procedencia de un gran número de personas. 
En el cuadro 1 se presentan los migrantes durante los quinquenios previos a los censos de 1960 a 1970 (los $G_{i j}$ para los periodos 1955-1960 y 1965-1970), y en la última parte la población media estimada para 1955, 1960, 1965 y 1970.

Estas poblaciones fueron estimadas de la siguiente manera. De los censos de 1950, 1960 y 1970 se obtuvo la proporción de población en cada una de las regiones para los años censales. Se supuso que estas distribuciones espaciales eran correctas, es decir, que no las afectaban coberturas censales diferenciales por región; supuesto que se hizo debido a que, en la literatura demográfica para México, no existen estudios que muestren el efecto distorsionador de coberturas diferenciales por región en la distribución espacial de la población. Suponiendo además que, el alza o la baja en la proporción de población en cada región entre 1950 y 1960 y entre 1960 y 1970 haya seguido una tendencia lineal, se obtuvieron las distribuciones espaciales para 1955 y 1965. Así por ejemplo, en los censos de población de 1950 y 1960 se captaron proporciones de población en la región 1 de 0.066885 y 0.074835 respectivamente; entonces, la proporción de población residente en la región 1 en 1955 fue estimada como $0.5 \cdot(0.066885+0.074835)=0.070860$. Finalmente, aceptando correctas las poblaciones medias estimadas por SPP, Conapo y Celade (1983) a nivel nacional para los años respectivos, se dedujo la población residente en cada región, multiplicando esos totales nacionales por la proporción respectiva.

Los operadores matriciales $\underset{\sim}{G}$ para los quinquenios $1955-1960$ y 1965-1970 se muestran en el cuadro 2.

¿Cómo obtener el operador $G$ para el quinquenio 1960-1965? Si bien una posibilidad hubiera sido trabajar con los flujos migratorios, captados por el censo de 1970 para ese periodo, ésta se desechó por un lado, debido al requerimiento de supuestos algo más complicados que para el quinquenio previo al censo, y por otro y fundamentalmente, a la posible ubicación errónea por parte del declarante del tiempo de su residencia en la entidad federativa en que fue entrevistado (redondeo a diez años antes del censo que lo ubicarían en el quinquenio 1955-1960).

El método utilizado fue el siguiente. Siendo la matriz $G$ de tipo probabilistico, una inicial para el quinquenio 1960-1965 fue estimada como la raíz cuadrada del producto de las correspondientes a los quinquenios previo y posterior. ${ }^{4}$ Los ocho elementos del $i$-ésimo (1967).

4 Acerca de potencias fraccionarias de matrices, véase Waugh y Abel 


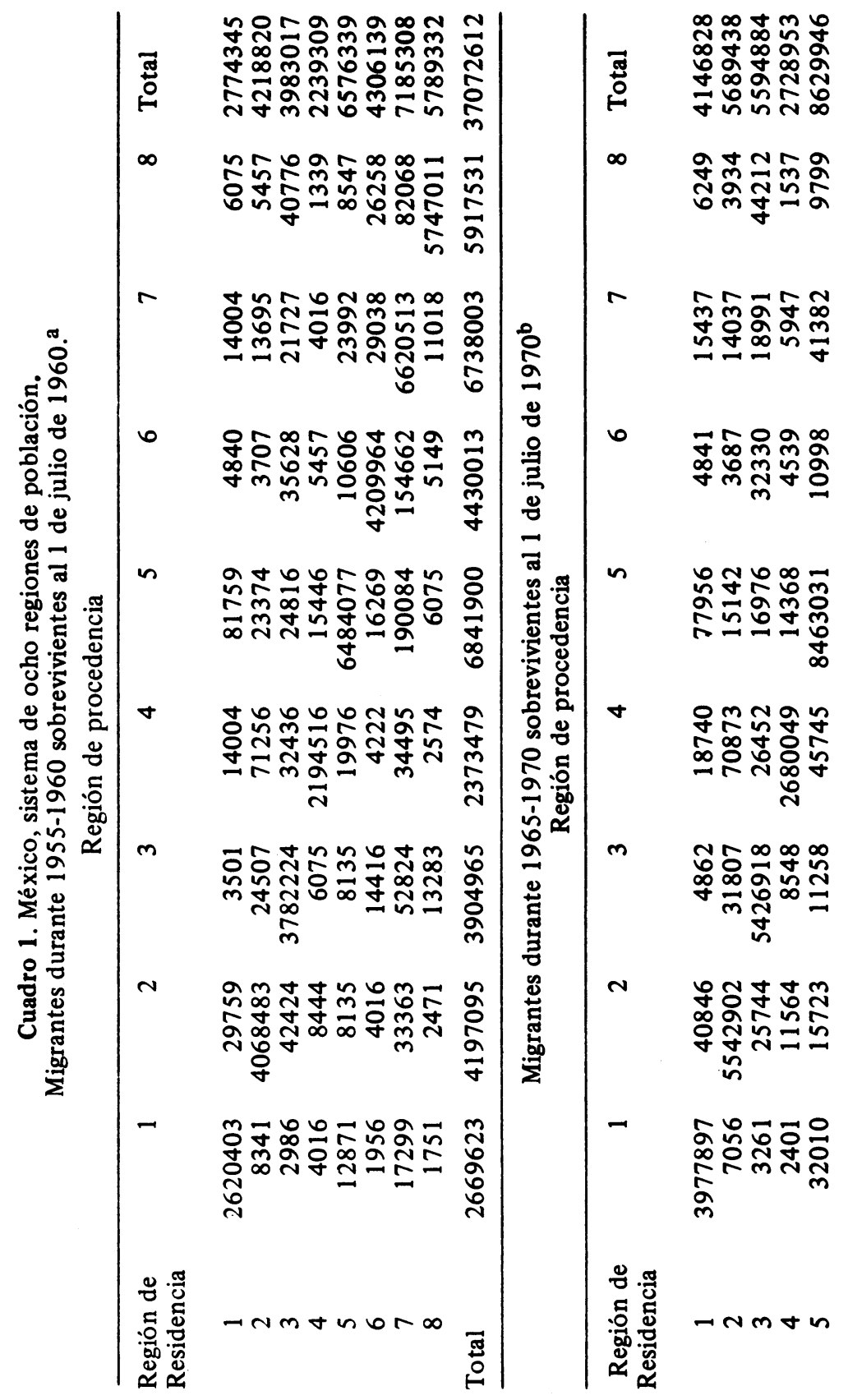




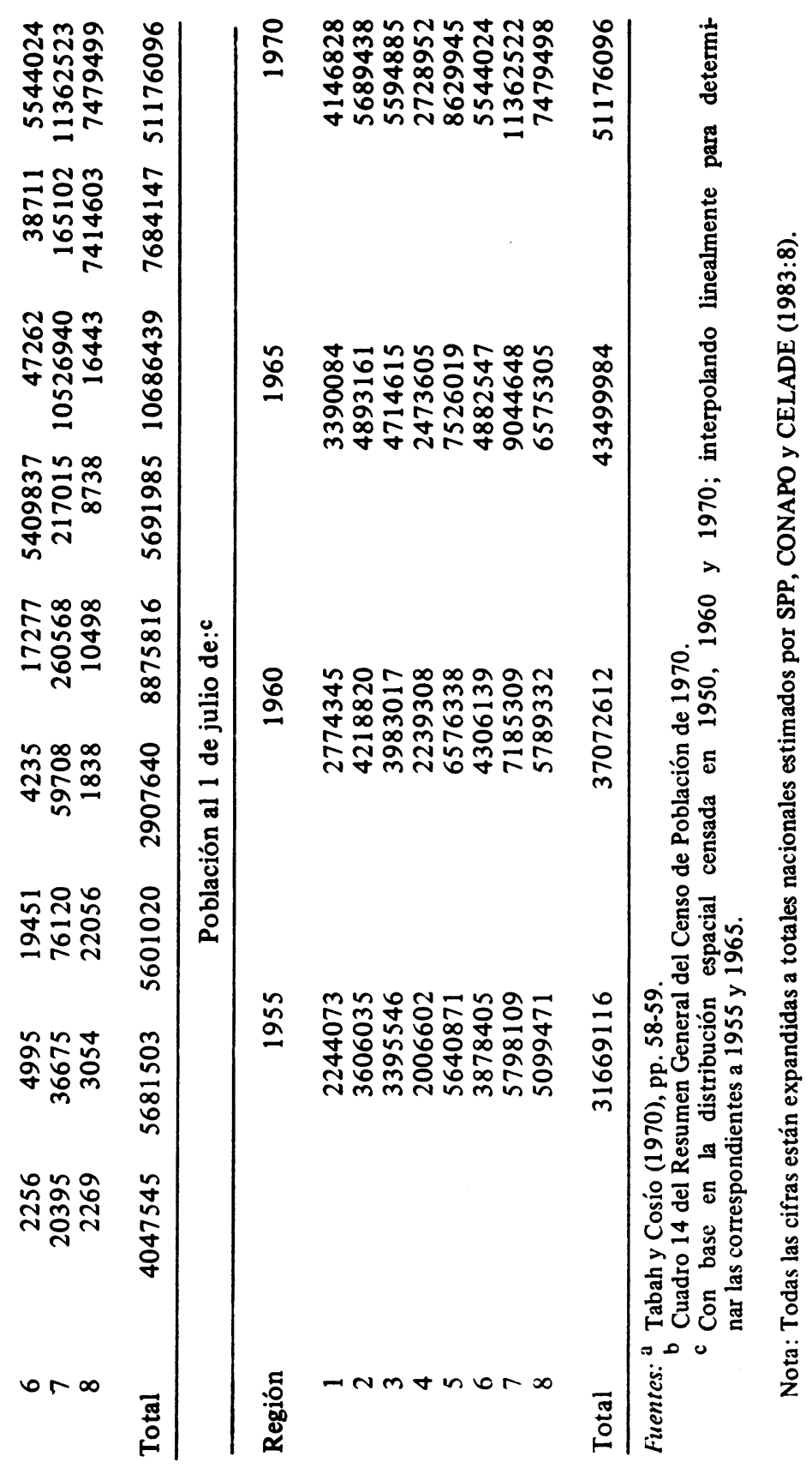




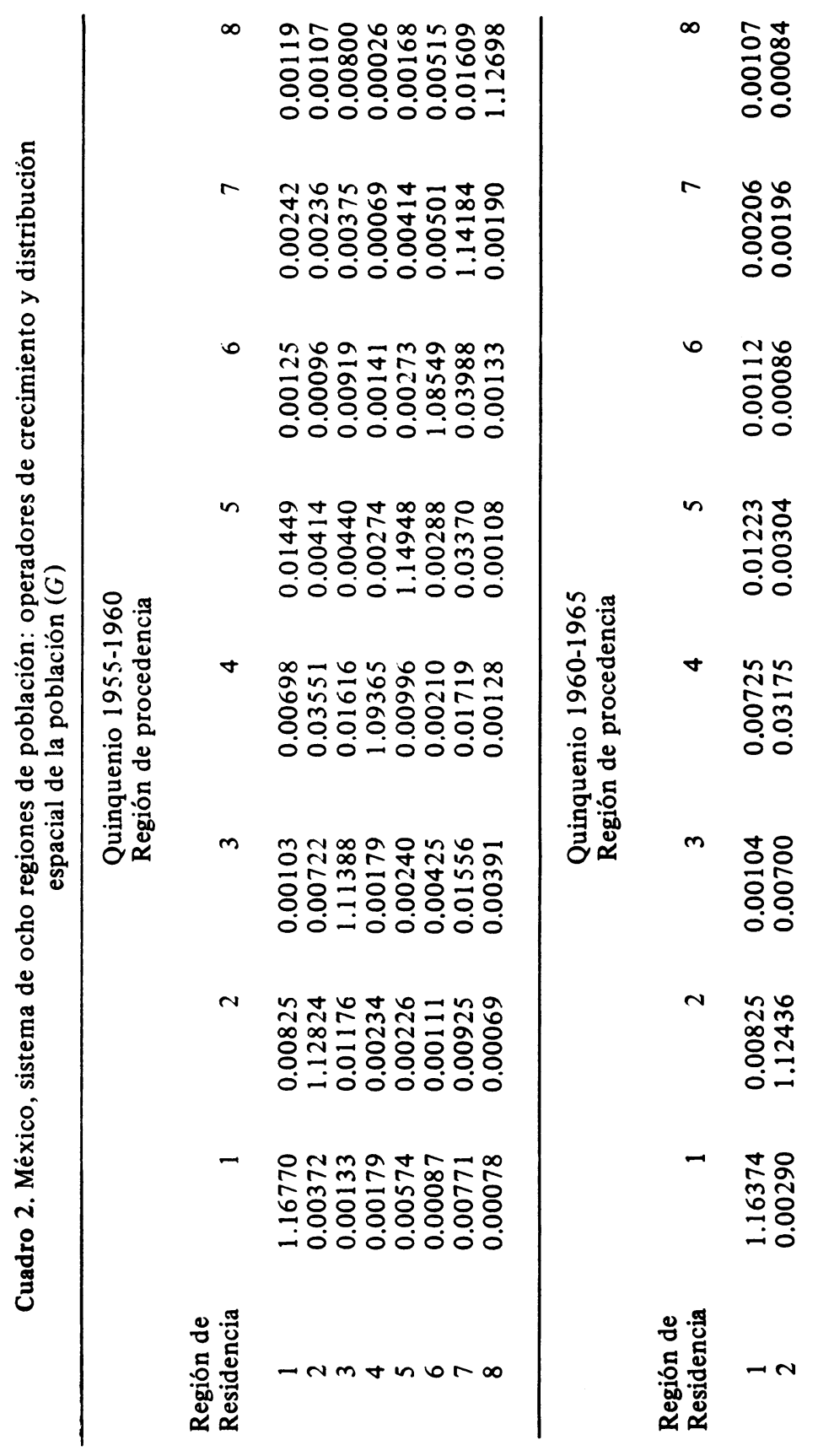




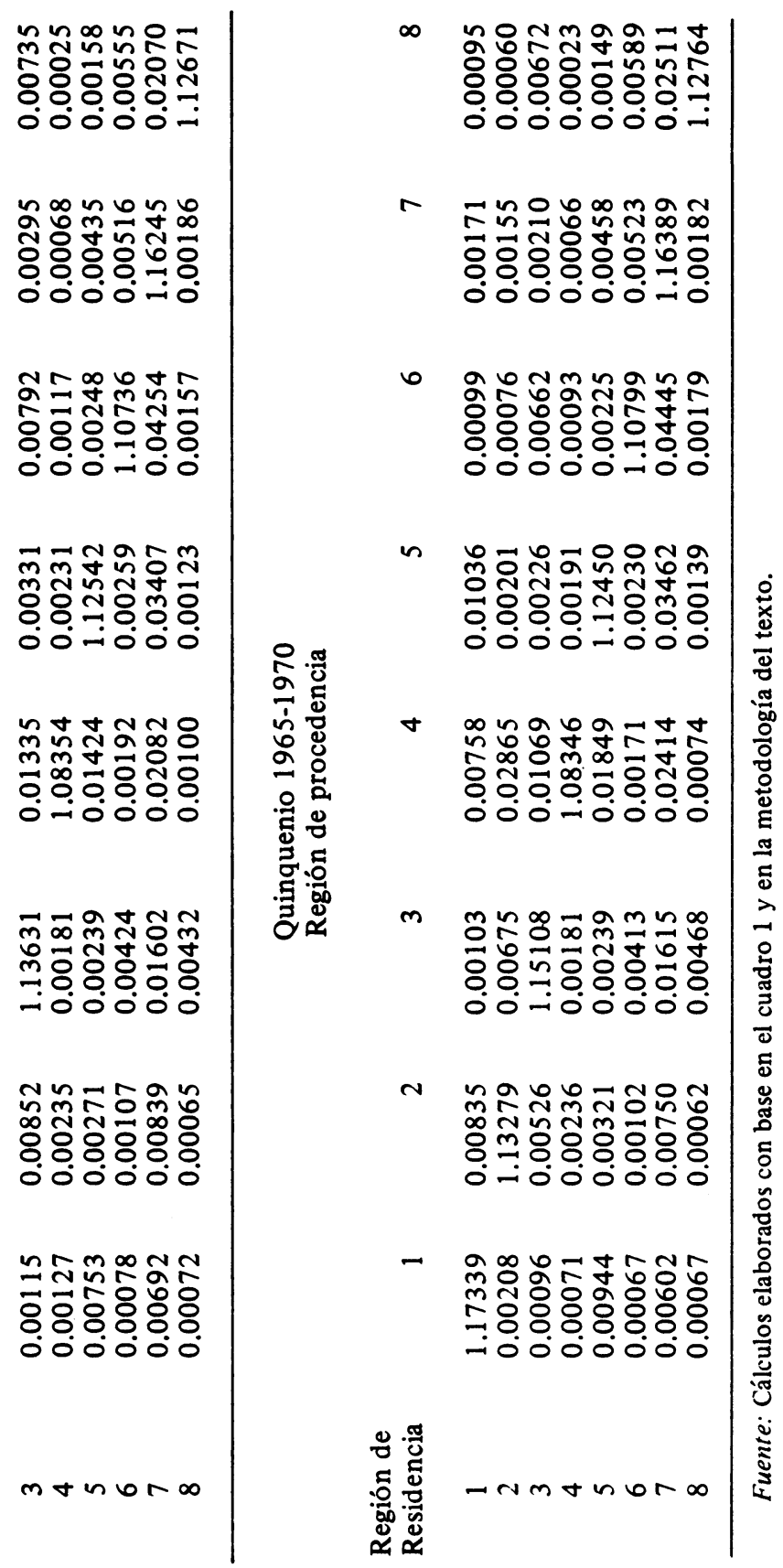


renglón de esa matriz inicial fueron entonces multiplicados por la proporción $N_{i}(1965) / N_{i}(1960)$, satisfaciendo de esa manera la ecuación (7). La matriz resultante, tomada como definitiva para el quinquenio 1960-1965, se muestra también en el cuadro 2 .

Un aspecto que conviene aclarar es el supuesto que la población del sistema multirregional está cerrada a la migración con cualquier región no considerada, lo que en el caso de México equivale a suponer una cerradura a la migración internacional. Con base en la información captada por los censos de 1970 de México y Estados Unidos, y considerando que la mayor parte de la emigración internacional mexicana es hacia ese país (Celade, 1977), se obtiene un total de 63991 inmigrantes internacionales hacia México durante el quinquenio 1965-1970 sobrevivientes al 1o. de julio de 1970, y de 186417 emigrantes mexicanos hacia Estados Unidos del mismo tipo. De estas cifras, se desprende un saldo neto migratorio negativo de 122426 durante el quinquenio 1965-1970 sobreviviente al 1o. de julio de 1970 para México, cifra por demás despreciable al compararla con los poco más de 51 millones de residentes en el país a mediados de 1970.

Finalmente, debido a que SPP, Conapo y Celade (1983) contemplan en sus estimaciones la ocurrencia de migración internacional en México durante el periodo 1955-1970, al aplicar el modelo multirregional para la migración interna del país, se está suponiendo que, los saldos netos externos negativos se distribuyen proporcionalmente en cada una de las ocho regiones, de acuerdo a la distribución espacial del tiempo vivido durante cada lustro.

\section{ANÁliSIS DE: los RESUltados ObTLinidos PARA MéXICO}

Una vez obtenidos los operadores $G$ para los tres quinquenios, mediante la aplicación de la fórmula $(\widetilde{8})$, se deducen las matrices $\underset{\sim}{\boldsymbol{M}}$ de tasas. Las tasas de emigración interregional obtenidas se presentan en el cuadro 5 y las de crecimiento natural en el cuadro 8. Obteniendo el tiempo vivido por la población en cada región mediante su aproximación lineal y multiplicando éste por las tasas respectivas, se determinan los crecimientos naturales absolutos y los flujos migratorios interregionales, resultados que se muestran en el cuadro 3.

Así, por ejemplo. los años-persona vividos por la población en la región 3 durante el quinquenio 1960-1965 se estimaron como 2.5 
Cuadro 3. México, sistema de ocho regiones de población: migrantes

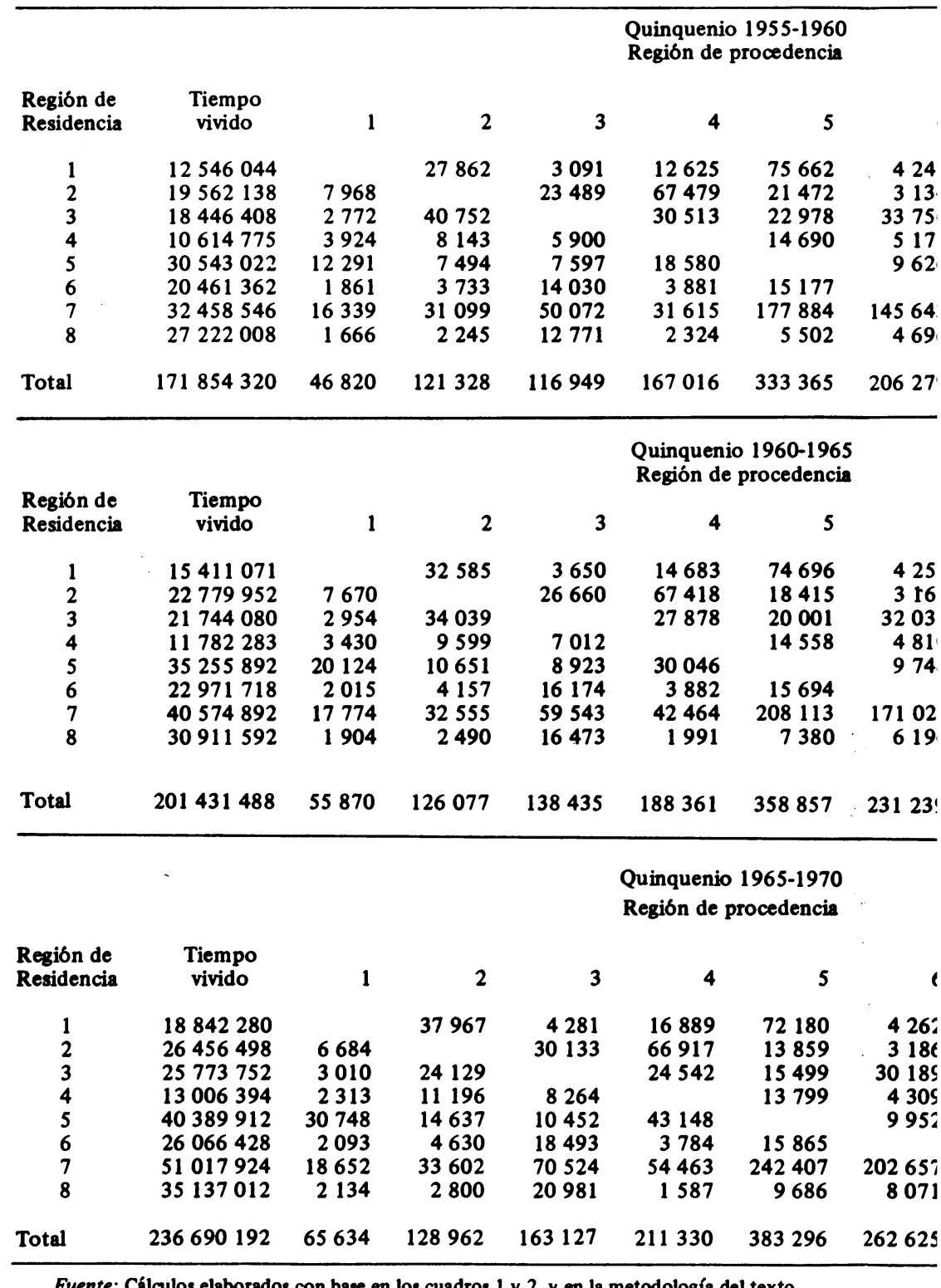

Fuente: Cálculos elaborados con base en los cuadros 1 y 2, y en la metodología del texto. 
na de ocho regiones de población: migrantes interregionales estimados.

\begin{tabular}{|c|c|c|c|c|c|c|c|}
\hline \multicolumn{8}{|c|}{$\begin{array}{l}\text { Quinquenio } 1955-1960 \\
\text { Región de procedencia }\end{array}$} \\
\hline 3 & 4 & 5 & 6 & 7 & 8 & Total & $\begin{array}{l}\text { Crec. } \\
\text { Nat. }\end{array}$ \\
\hline $\begin{array}{r}3091 \\
23489 \\
\\
5900 \\
7597 \\
14030 \\
50072 \\
12771\end{array}$ & $\begin{array}{r}12625 \\
67479 \\
30513 \\
18580 \\
3881 \\
31615 \\
2324\end{array}$ & $\begin{array}{r}75662 \\
21472 \\
22978 \\
14690 \\
\\
15177 \\
177884 \\
5502\end{array}$ & $\begin{array}{r}4245 \\
3134 \\
33756 \\
5178 \\
9626 \\
145643 \\
4696\end{array}$ & $\begin{array}{r}13259 \\
13233 \\
21167 \\
3916 \\
23205 \\
28999 \\
10752\end{array}$ & $\begin{array}{r}5436 \\
4878 \\
38432 \\
1189 \\
7737 \\
25002 \\
76017\end{array}$ & $\begin{array}{r}142179 \\
141653 \\
190368 \\
42939 \\
86530 \\
92684 \\
528668 \\
39957\end{array}$ & $\begin{array}{r}434913 \\
592460 \\
514052 \\
356783 \\
1182302 \\
541329 \\
973061 \\
808595\end{array}$ \\
\hline 116949 & 167016 & 333365 & 206279 & 114530 & 158691 & 1264978 & 5403496 \\
\hline \multicolumn{8}{|c|}{$\begin{array}{l}\text { Quinquenio } 1960-1965 \\
\text { Región de procedencia }\end{array}$} \\
\hline 3 & 4 & 5 & 6 & 7 & 8 & Total & $\begin{array}{l}\text { Crec. } \\
\text { Nat. }\end{array}$ \\
\hline $\begin{array}{r}3650 \\
26660 \\
\\
7012 \\
8923 \\
16174 \\
59543 \\
16473\end{array}$ & $\begin{array}{r}14683 \\
67418 \\
27878 \\
\\
30046 \\
3882 \\
42464 \\
1991\end{array}$ & $\begin{array}{r}74696 \\
18415 \\
20001 \\
14558 \\
15694 \\
208113 \\
7380\end{array}$ & $\begin{array}{r}4257 \\
3167 \\
32032 \\
4810 \\
9748 \\
171029 \\
6196\end{array}$ & $\begin{array}{r}14011 \\
13639 \\
20409 \\
4787 \\
30556 \\
36638 \\
13066\end{array}$ & $\begin{array}{r}5539 \\
4299 \\
39706 \\
1288 \\
8301 \\
30237 \\
110237\end{array}$ & $\begin{array}{r}149421 \\
141269 \\
177018 \\
45484 \\
118350 \\
108797 \\
641714 \\
49500\end{array}$ & $\begin{array}{r}522189 \\
659148 \\
693015 \\
377174 \\
1190187 \\
698849 \\
1350732 \\
936079\end{array}$ \\
\hline 138435 & 188361 & 358857 & 231239 & 133108 & 199607 & 1431554 & 6427375 \\
\hline \multicolumn{8}{|c|}{$\begin{array}{l}\text { Quinquenio } 1965-1970 \\
\text { Región de procedencia }\end{array}$} \\
\hline 3 & 4 & 5 & 6 & 7 & 8 & Total & $\begin{array}{l}\text { Crec. } \\
\text { Nat. }\end{array}$ \\
\hline $\begin{array}{r}4281 \\
30133 \\
8264 \\
10452 \\
18493 \\
70524 \\
20981\end{array}$ & $\begin{array}{r}16889 \\
66917 \\
24542 \\
\\
43148 \\
3784 \\
54463 \\
1587\end{array}$ & $\begin{array}{l}72180 \\
13859 \\
15499 \\
13799\end{array}$ & $\begin{array}{r}4262 \\
3186 \\
30189 \\
4309 \\
9952\end{array}$ & $\begin{array}{r}14512 \\
13518 \\
18108 \\
5857 \\
40408 \\
46620 \\
\\
16002\end{array}$ & $\begin{array}{r}5562 \\
3421 \\
41011 \\
1368 \\
8814 \\
36428 \\
152071\end{array}$ & $\begin{array}{r}155653 \\
137717 \\
156489 \\
47106 \\
158158 \\
127914 \\
774375 \\
61262\end{array}$ & $\begin{array}{r}666726 \\
787522 \\
886909 \\
419571 \\
1329064 \\
796189 \\
1698525 \\
1091605\end{array}$ \\
\hline 163127 & 211330 & 383296 & 262625 & 155026 & 248674 & 1618674 & 7676110 \\
\hline
\end{tabular}

2, y en la metodología del texto. 
$\left[N_{3}(1960)+N_{3}(1965)\right]=2.5(3983027+4714615)=21744080$. Siendo la tasa de emigración desde la región 3 hasta la 4 estimada para ese quinquenio de 0.000322 (cuadro 5), entonces el flujo migratorio estimado de la 3 hacia la 4 es de $(0.000322)(21744080)=7012 ; y$ siendo la tasa de crecimiento natural en la región 3 estimada para ese quinquenio de $\mathbf{0 . 0 3 1 8 7}$, entonces el crecimiento natural absoluto estimado para la región entre 1960 y 1965 es de $(0.03187)$ $(21744080)=693015$. Estas cifras se pueden corroborar en las casillas respectivas del cuadro 3.5

Un primer aspecto que salta a la vista es que, para los quinquenios 1955-1960 y 1965-1970, los flujos interregionales sobrevivientes al final de esos lustros (cuadro 1) resultan mayores que los estimados ocurridos durante los quinquenios (cuadro 3), para todas las corrientes. La explicación más probable de este rasgo es que la reproducción de los migrantes en la región de su destino, supere en monto a las defunciones de los migrantes.

En las cifras del cuadro 3, se podrá notar que, si bien el total de migraciones interregionales en el paŕs mostró aumentos de $13.2 \%$ del primero al segundo quinquenios y de $13.1 \%$ del segundo al tercero, los del número de años-persona vividos a nivel nacional, $17.2 \%$ del primero al segundo quinquenios y $17.5 \%$ del segundo al tercero (equivalente a los aumentos en la población expuesta al riesgo de migrar interregionalmente), fueron mayores; de donde, se puede concluir, como también se verá más adelante, que la intensidad de la migración entre las ocho regiones consideradas disminuyó con el paso del tiempo, entre 1955 y 1970.

La región que mayor cantidad de población expulsó durante los tres quinquenios fue la 5 u Occidente $(333365,358857$ y 382296 respectivamente), resultado de importantes flujos migratorios hacia las regiones 1 o Noroeste y 7 o Centro-Sur. Las emigraciones desde la región 5 hacia la 1 representaron $22.7,20.8$ y 18.8 por ciento de la expulsión total de la región para los tres quinquenios respectivamente, y sus salidas hacia las 7 representaron $53.4,58.0$ y 63.2 por ciento. Si tomamos en cuenta que Guanajuato, Jalisco y Michoacán son tres de los cuatro estados de la región Occidente (lo que comprende la inmensa mayoría de la población de la zona), las cifras

5 Si el lector realiza los dos productos de las tasas por el tiempo vivido, notará que los resultados difieren ligeramente de los mostrados en el cuadro 3. Esto se debe a que los cálculos fueron hechos en una computadora, donde, para las tasas, se consideraron más decimales que las mostradas en el texto y en los cuadros 5 y 8 . 
anteriores reflejan los conocidos flujos migratorios desde esas tres entidades federativas hacia la frontera noroeste del país y hacia la ciudad de México. 6

Otras expulsiones totales importantes fueron las ocurridas desde las regiones 6 y 8, en su mayor parte dirigidas hacia la región 7 , y de éstas casi su totalidad con destino a la ciudad de México, y desde la región 4, las principales se dirigieron hacia las regiones 2,7 y 5 .

Se podrán notar, en el otro extremo, algunos flujos bastante pequeños, como los de la región 8 hacia la 4 , y de las regiones 1 y 2 hacia la 8 . El bajo monto de estas dos últimas, si bien se ha debido en parte a la lejanía que separa a las regiones Noroeste y Norte de la Sureste, es más probable que se haya debido a que las dos primeras regiones ofrecían mejores perspectivas de vida que la tercera.

Del lado de la inmigración, la corriente dirigida hacia la región 7 , y nuevamente en su inmensa mayoría hacia la ciudad de México, fue la más voluminosa del flujo interregional total nacional; provino de las regiones Occidente, Centro y Sureste $(5,6$ y 8$)$, en su conjunto, 75.6, 76.3 y 77.1 por ciento del total de entrantes a la región CentroSur, para los tres quinquenios, respectivamente.

En el cuadro 4 se presenta la distribución porcentual de los diferentes flujos interregionales con respecto al total nacional. El análisis mostrado arriba se reproduce exactamente en estas cifras. La mayor parte de las emigraciones interregionales en el país se dieron, en orden de importancia, desde las regiones 5,6 y 8 para los tres quinquenios; y casi la mitad de las migraciones interregionales en todo el país tuvieron su destino en la región 7 (en su gran mayoría en la ciudad de México). En orden de importancia de las inmigraciones, se puede ver que, si bien durante los dos primeros quinquenios, la región 3 ocupó el segundo lugar, detrás de la región 7, en el tercero, ocupaba el tercer lugar.

Pero la región 5, que ocupó el sexto y quinto lugar en los dos primeros quinquenios, pasó a ocupar el segundo lugar en el tercero, debido probablemente al retorno hacia ella desde las regiones 1 y 2 . Este hecho se puede ver en el incremento en los porcentajes que, con respecto al total nacional, representaron las corrientes desde la Noro-

6 La zona metropolitana de la ciudad de México congregaba $77.6 \%$ de la población de la región 7 en 1960 y $80.8 \%$ en 1970. Por zona metropolitana de la ciudad de México se considera aquí el espacio geográfico comprendido por el Distrito Federal y los municipios de Atizapán de Zaragoza, Coacalco, Cuautitlán, Chimalhuacán, Ecatepec, Huixquilucan, Naucalpan, Netzahualcóyotl, La Paz, Tlalnepantla y Tultitlán del estado de México. 
Cuadro 4. México, sistema de ocho regiones de población: distribución porcentual de los flujos migratorios

Quinquenio 1955-1960

Región de procedencia

\begin{tabular}{crrrrrrrrr}
$\begin{array}{c}\text { Región de } \\
\text { Residencia }\end{array}$ & 1 & 2 & 3 & 4 & 5 & 6 & 7 & 8 & Total \\
1 & & 2.2 & 0.2 & 1.0 & 6.0 & 0.3 & 1.0 & 0.4 & 11.2 \\
2 & 0.6 & & 1.9 & 5.3 & 1.7 & 0.2 & 1.0 & 0.4 & 11.2 \\
3 & 0.2 & 3.2 & & 2.4 & 1.8 & 2.7 & 1.7 & 3.0 & 15.0 \\
4 & 0.3 & 0.6 & 0.5 & & 1.2 & 0.4 & 0.3 & 0.1 & 3.4 \\
5 & 1.0 & 0.6 & 0.6 & 1.5 & & 0.8 & 1.8 & 0.6 & 6.8 \\
6 & 0.1 & 0.3 & 1.1 & 0.3 & 1.2 & & 2.3 & 2.0 & 7.3 \\
7 & 1.3 & 2.5 & 4.0 & 2.5 & 14.1 & 11.5 & & 6.0 & 41.8 \\
8 & 0.1 & 0.2 & 1.0 & 0.2 & 0.4 & 0.4 & 0.8 & & 3.2 \\
Total & 3.7 & 9.6 & 9.2 & 13.2 & 26.4 & 16.3 & 9.1 & 12.5 & 100.0 \\
\hline
\end{tabular}

Quinquenio 1960-1965

Región de procedencia

$\begin{array}{crrrrrrrrr}\begin{array}{c}\text { Región de } \\ \text { Residencia }\end{array} & 1 & 2 & 3 & 4 & 5 & 6 & 7 & 8 & \text { Total } \\ 1 & & 2.3 & 0.3 & 1.0 & 5.2 & 0.3 & 1.0 & 0.4 & 10.4 \\ 2 & 0.5 & & 1.9 & 4.7 & 1.3 & 0.2 & 1.0 & 0.3 & 9.9 \\ 3 & 0.2 & 2.4 & & 1.9 & 1.4 & 2.2 & 1.4 & 2.8 & 12.4\end{array}$


Cuadro 4. México, sistema de ocho regiones de población: distribución porcentual de los flujos migratorios (conclusión)

\begin{tabular}{rrrrrrrrrr}
\hline 4 & 0.2 & 0.7 & 0.5 & & 1.0 & 0.3 & 0.3 & 0.1 & 3.2 \\
5 & 1.4 & 0.7 & 0.6 & 2.1 & & 0.7 & 2.1 & 0.6 & 8.3 \\
6 & 0.1 & 0.3 & 1.1 & 0.3 & 1.1 & & 2.6 & 2.1 & 7.6 \\
7 & 1.2 & 2.3 & 4.2 & 3.0 & 14.5 & 11.9 & & 7.7 & 44.8 \\
8 & 0.1 & 0.2 & 1.2 & 0.1 & 0.5 & 0.4 & 0.9 & & 3.5 \\
& & & & & & & & & \\
Total & 3.9 & 8.8 & 9.7 & 13.2 & 25.1 & 16.2 & 9.3 & 13.9 & 100.0 \\
\hline
\end{tabular}

Quinquenio 1965-1970

Región de procedencia

\begin{tabular}{crrrrrrrrr}
$\begin{array}{c}\text { Región de } \\
\text { Residencia }\end{array}$ & 1 & 2 & 3 & 4 & 5 & 6 & 7 & 8 & Total \\
1 & & 2.3 & 0.3 & 1.0 & 4.5 & 0.3 & 0.9 & 0.3 & 9.6 \\
2 & 0.4 & & 1.9 & 4.1 & 0.9 & 0.2 & 0.8 & 0.2 & 8.5 \\
3 & 0.2 & 1.5 & & 1.5 & 1.0 & 1.9 & 1.1 & 2.5 & 9.7 \\
4 & 0.1 & 0.7 & 0.5 & & 0.9 & 0.3 & 0.4 & 0.1 & 2.9 \\
5 & 1.9 & 0.9 & 0.6 & 2.7 & & 0.6 & 2.5 & 0.5 & 9.8 \\
6 & 0.1 & 0.3 & 1.1 & 0.2 & 1.0 & & 2.9 & 2.3 & 7.9 \\
7 & 1.2 & 2.1 & 4.4 & 3.4 & 15.0 & 12.5 & & 9.4 & 47.8 \\
8 & 0.1 & 0.2 & 1.3 & 0.1 & 0.6 & 0.5 & 1.0 & & 3.8 \\
Total & 4.1 & 8.0 & 10.1 & 13.1 & 23.7 & 16.2 & 9.6 & 15.4 & 100.0 \\
\hline
\end{tabular}

Fuente: Cálculos elaborados con base en el cuadro 3. 
este hacia la Occidente $(1.0,1.4$ y $1.9 \%)$ y desde la Norte hacia la Occidente $(0.6,0.7$ y $0.9 \%)$. El mismo fenómeno se ve en el cuadro 3, donde el primero de esos flujos se incrementó en $63.7 \%$ del primero al segundo quinquenio y en $52.8 \%$ del segundo al tercero, y la segunda de esas corrientes en $\mathbf{4 2 . 1}$ y 37.4 por ciento respectivamente; ambos por encima de los observados para el total nacional (13.2 y $13.1 \%$ ). Es más, el flujo total de emigrantes desde la región 1 , hacia la 5 pasó de representar 26.3\% durante el primer quinquenio, a 36.0 y 46.8 por ciento en los dos siguientes, y el total de emigrantes de la región 2 hacia la 5 de $6.2 \%$ en el primer lustro, a 8.4 y 11.3 por ciento en los dos posteriores. 0 bien, de la inmigración total hacia la Occidente, los desplazamientos originados en la Noroeste y en la Norte, en su conjunto, pasaron de representar $22.9 \%$ en el primer quinquenio, a 26.0 y el 28.7 por ciento en los dos siguientes.

En los resultados que se presentan en el cuadro 8 , se puede ver el descenso que hubo en la intensidad de la migración interregional total en México con el paso del tiempo, entre 1955 y 1970. La tasa bruta nacional descendió de 7.36 por mil entre 1955 y 1960 a 7.11 y 6.84 en los dos quinquenios siguientes.

En las tasas de emigración que se presentan en el cuadro 5 , al nivel de tasa global para las regiones, se puede ver que la región Centro-Norte (4) mostraba la mayor intensidad, y creciente con el paso de los años, entre las ocho consideradas. La región Occidente (5), que ocupaba el segundo lugar en los dos primeros quinquenios, pasó a ocupar el tercero en el último periodo, intercambiando lugares con la Centro (6), aunque la primera mostró una tendencia descendente y la segunda se mantuvo constante. La región Sureste (8), que ocupaba el sexto lugar en cuanto a intensidad, ascendió en tal forma, que para el periodo 1965-1970 se encontraba ya en cuarto lugar.

Al nivel de intercambios interregionales, la emigración más intensa, y creciente, a lo largo del periodo de quince años, fue la originada en la región 6 dirigida hacia la 7 ;y muy cerca de ella estuvieron la de la 5 hacia la 7 , con tendencia creciente, y la de la 4 hacia la 2, con tendencia decreciente. La fuerte atracción que la región 7 , y principalmente la ciudad de México, ejerce sobre las restantes siete regiones (prácticamente el resto del país), queda evidenciada en las tasas de emigración de las restantes regiones hacia ella, superiores a uno por mil en los tres quinquenios (con la ligera excepción del flujo procedente de la 1 en el último quinquenio).

Este último fenómeno se puede apreciar también en las tasas de inmigración que se presentan en el cuadro 6. La intensidad del flujo inmigratorio total hacia la Centro-Sur fue la mayor, aunque con ten- 


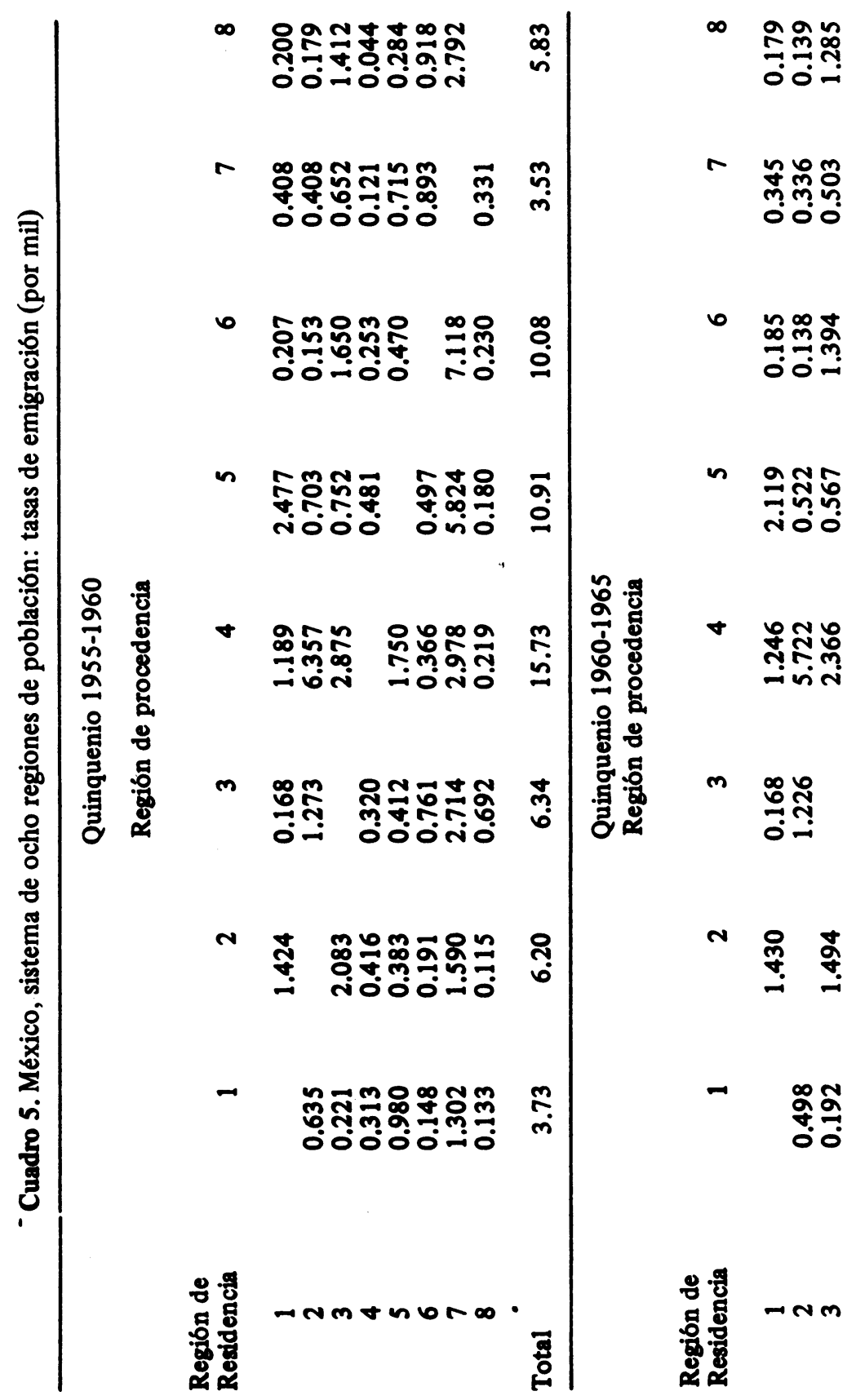




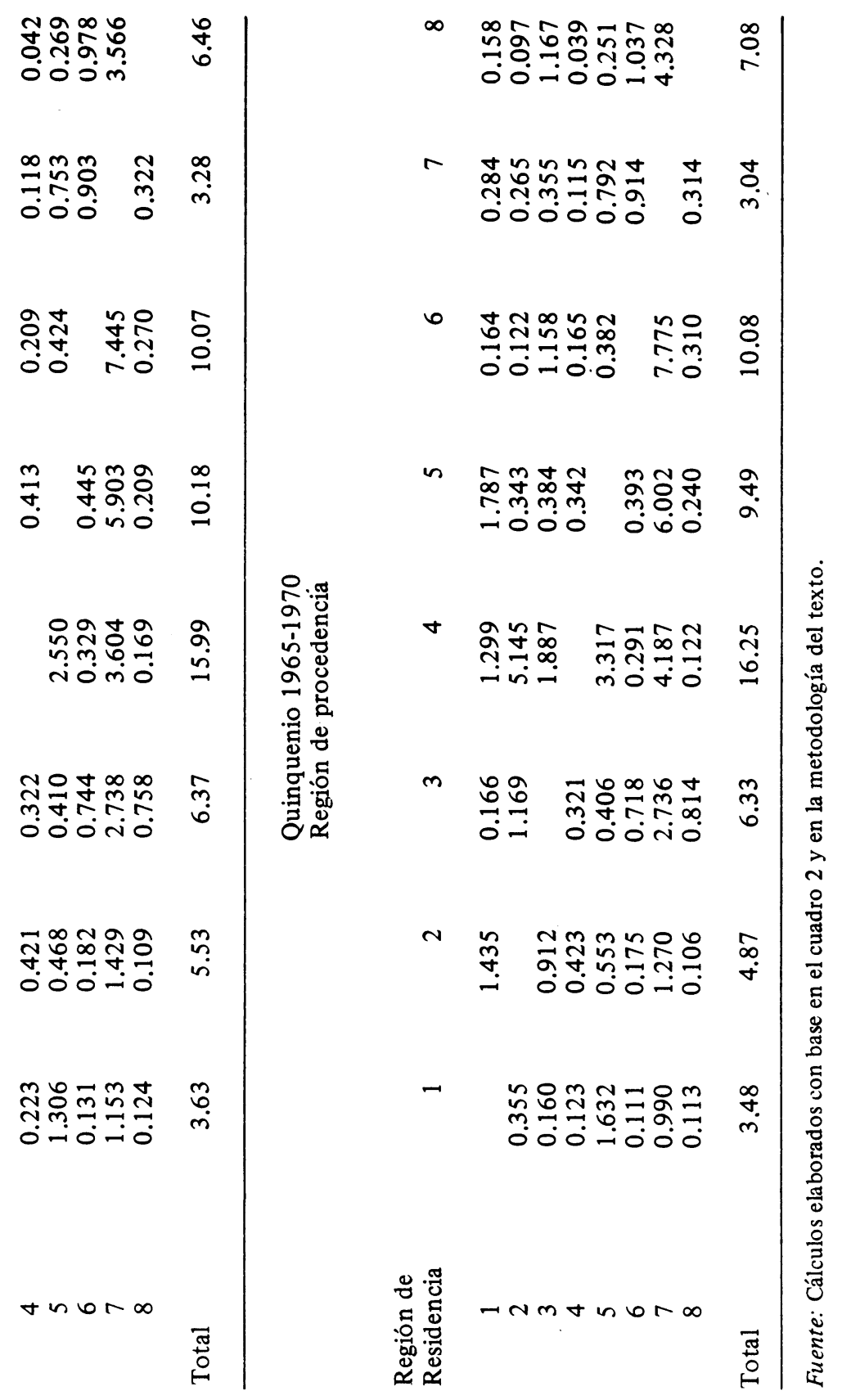


dencia ligeramente decreciente, en los tres quinquenios, llegando a ser incluso, en los dos últimos lustros considerados, 63 y $84 \%$ superior a la siguiente en importancia (la inmigración total hacia la región 1).

La fuerte intensidad de la inmigración hacia la región 7 en $75.6,76.2$ y $77.1 \%$ para los tres quinquenios, respectivamente se debió a los movimientos originados en las regiones Occidente (5), Centro (6) y Sureste (8); sensiblemente descendentes las tendencias en las dos primeras corrientes y ascendente en la tercera. La intensidad de los flujos de las regiones 5 y 6 hacia la 7 se encontraban entre las tres más altas, siendo la restante la de la 5 hacia la 1 , aunque esta última notablemente descendente, se redujo $36.5 \%$ del primero al tercer quinquenio.

Incluso la posibilidad mencionada del retorno de la frontera norte del país (regiones 1 y 2) hacia la Occidente, se puede ver también en lo siguiente. Si no hubiera habido migración hacia la 5 desde la 1 y la 2, la tasa de inmigración total en la Occidente se habría incrementado $13.6 \%$ del primero al segundo quinquenios y $12.4 \%$ del segundo al tercero; los aumentos reales fueron de 18.7 y 16.7 respectivamente, y aumento la tasa de los flujos procedentes de las regiones 1 y 2 en su conjunto hacia la 5 en 34.9 y $28.6 \%$.

La contribución neta de la migración al crecimiento demográfico de las regiones puede verse en el cuadro 7. Sólo la región Noreste (3) mostró cambio de signo (de positivo a negativo) al cabo de los tres quinquenios, y sólo las regiones Centro-Norte (4) y Sureste (8) no mostraron tendencias decrecientes en su ganancia o pérdida neta por migración.

Las mayores tasas de migración neta positiva total fueron las observadas en la región 7 , que mostraron una tendencia decreciente, debido a un descenso poco más acelerado en su inmigración relativa (cuadro 6) que en su emigración (cuadro 5). Este descenso, a nivel interregional, fue el resultado de una mayor disminución en sus altas tasas de ganancia con respecto a las regiones 5 y 6 , que los aumentos en las correspondientes a su intercambio con las regiones 3,4 y 8 .

La región Centro-Norte (4), en cambio, mostró las tasas negativas más altas, exhibiendo una tendencia creciente, pues a pesar de descender sus pérdidas netas relativas con respecto a las regiones 2 y 3 , los aumentos en las correspondientes a su intercambio con las regiones 5 y 7 se dieron con mayor intensidad.

La región Occidente (5) mostró la mayor reducción en la tasa neta negativa total ( $31 \%$ del primer al tercer quinquenios), resultado fundamentalmente de las abruptas reducciones en el intercambio con 


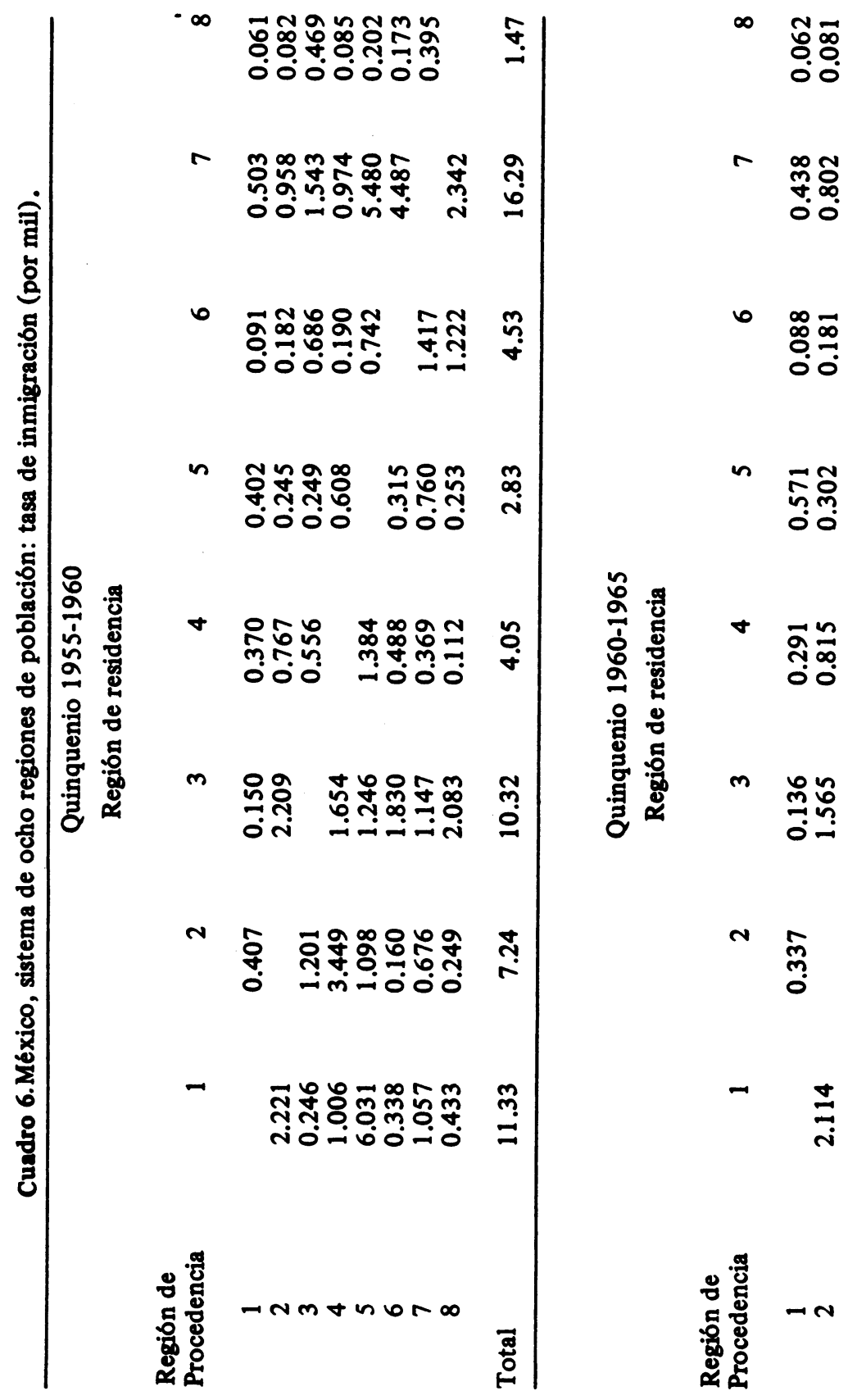




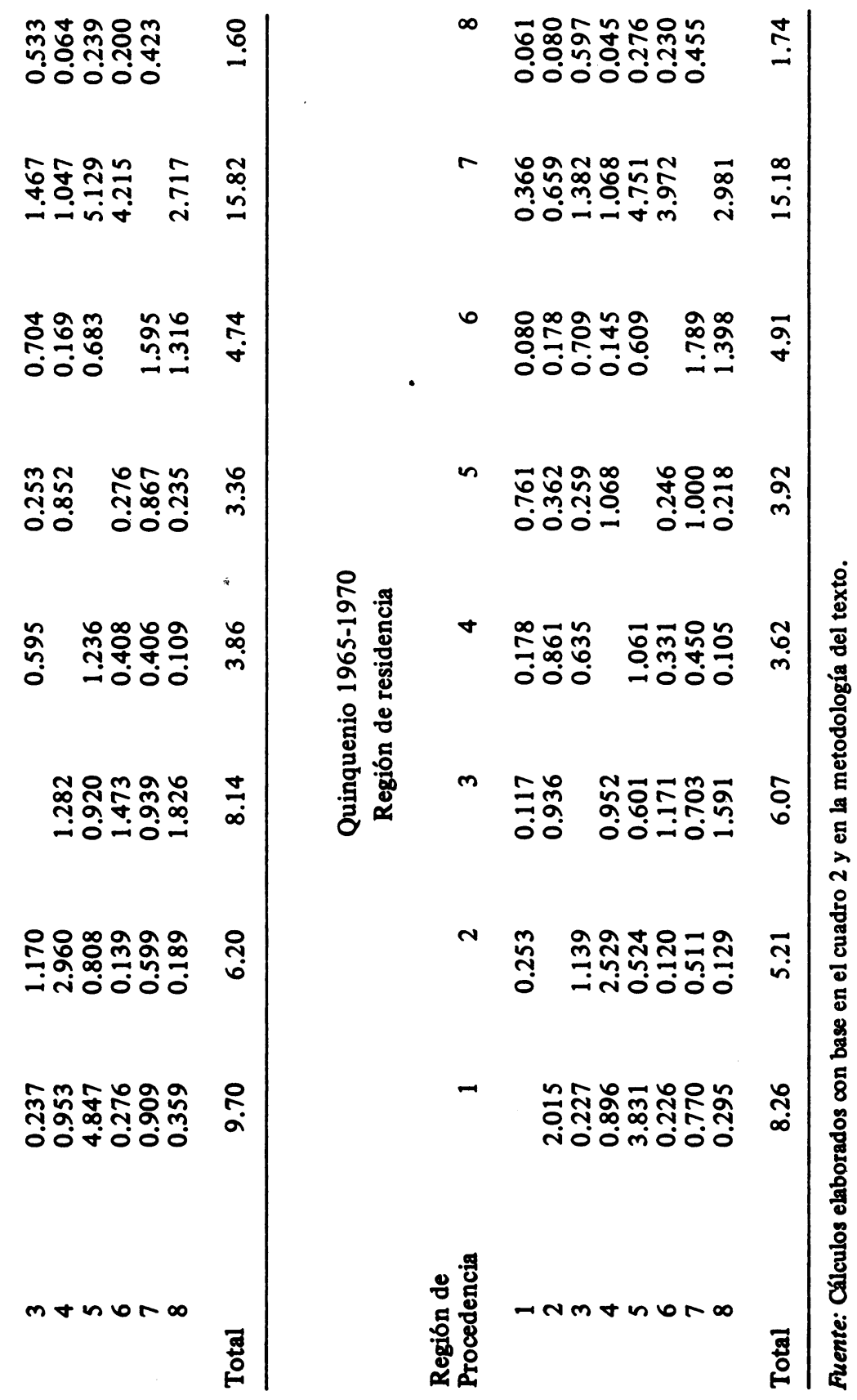




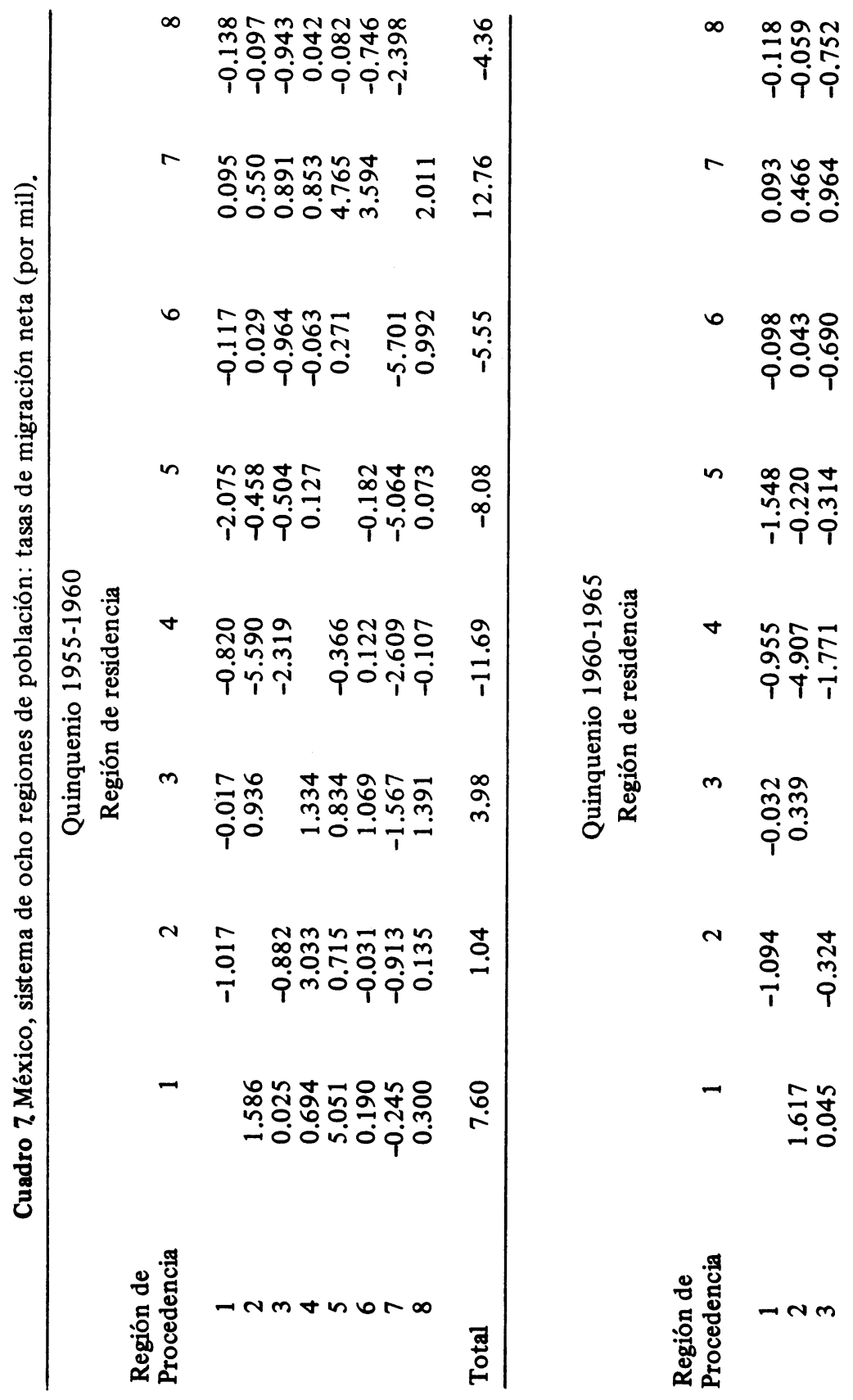




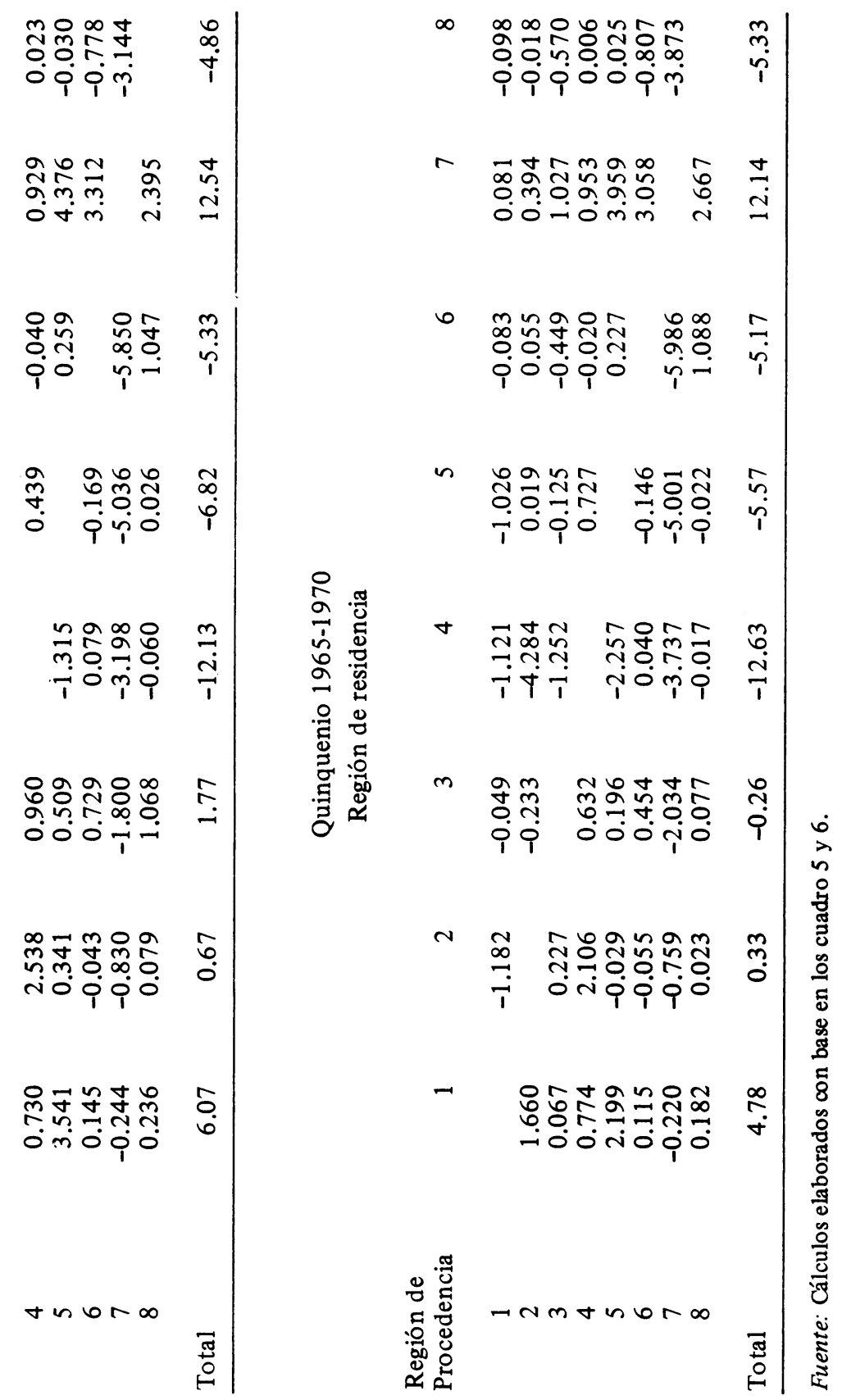




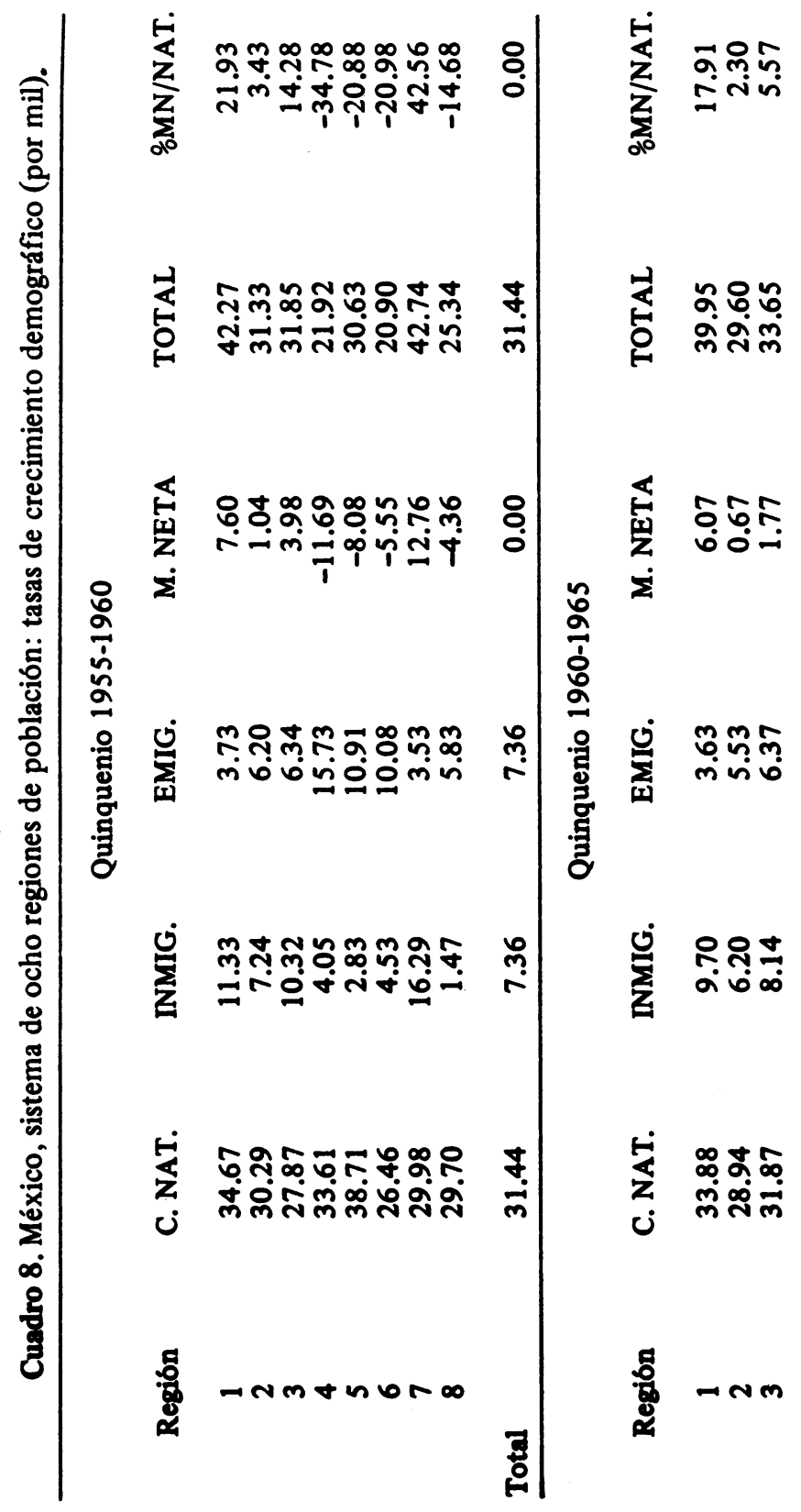




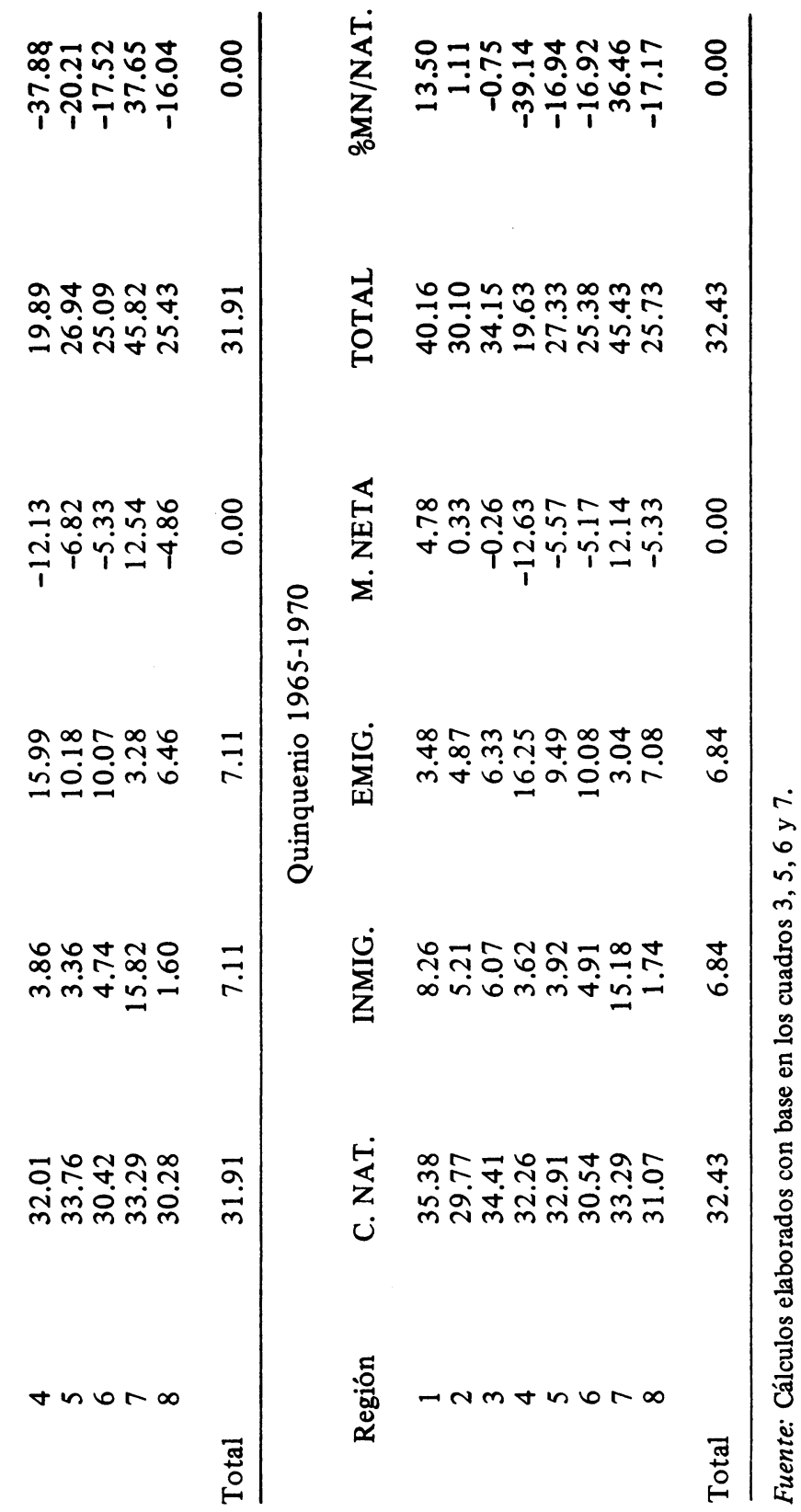


la Noroeste $(50.6 \%$ de reducción de la tasa de migración neta del primer al tercer quinquenios) y con la Norte, donde de negativa pasó a positiva.

La región Norte (2), en cambio, exhibió la mayor reducción en la tasa neta positiva (68.3\% del primer al tercer quinquenios), resultado del fuerte descenso de su ganancia con respecto a la región 4 $(30.6 \%)$, y con respecto a la 5 , pasando incluso ésta de positiva a negativa de 1955-1960 a 1965-1970.

La región Noreste (1), también exhibió un fuerte descenso en su tasa neta positiva (37.1\% del primer al tercer quinquenios), resultado de la abrupta disminución en su ganancia neta con respecto a la Occidente $(56.5 \%)$, no obstante que aumentaron sus tasas netas positivas con respecto a las regiones 2,3 y 4 y disminuyó su negativa con respecto a la 7 .

Se podrá ver, finalmente, que si bien ninguna región exhibió tasas negativas con respecto a las siete regiones restantes, sólo la Centro-Sur ( 7 y principalmente la ciudad de México) mostró tasas positivas con respecto a todas las demás $\mathrm{y}$, consecuentemente, todas éstas tuvieron tasas negativas con respecto a la 7.

En el cuadro 8 se presentan las componentes estimadas del crecimiento demográfico total en las ocho regiones y para los tres quinquenios.

Se puede ver, en primer lugar, un fuerte y ascendente crecimiento natural nacional, resultado de una alta y sostenida fecundidad y una mortalidad en descenso. $Y$, mientras durante el quinquenio 1955-1960, cuatro regiones lo exhibieron por debajo de 30 por mil, diez años después sólo una-la Norte-, aunque levemente por debajo, se encontró en tal caso.

Los menores crecimientos naturales o vegetativos en las regiones 6 y 8 , en los tres quinquenios, pueden deberse a una mayor mortalidad, si consideramos que estas dos regiones se encontraban entre las menos prósperas del país, económicamente hablando. ${ }^{7}$

El bajo crecimiento natural en la región Norte, sin embargo, si bien pudo deberse a una menor fecundidad, es más probable que aparezca por deficiencias del modelo, ya que el crecimiento vegetativo proviene fundamentalmente de la diagonal principal del operador matricial $G$. Con todo, los errores que se puedan tener en las estimaciones, no parecen ser tan significativos, como para modificar las

7 A este respecto, véase Unikel, et al. (1978), pp. 68-74. 
conclusiones de la contribución del crecimiento social o migración neta al crecimiento total de las regiones. ${ }^{8}$

En la región Centro-Sur (7), la fuerte inmigración, que produjo altas tasas de migración neta positiva, propició que su crecimiento social haya incrementado $42.6,37.7$ y $36.5 \%$, durante los tres quinquenios, respectivamente (última columna del cuadro 8 ), su crecimiento natural. $\mathrm{O}$ bien, en otros términos, que haya contribuido con $30.0,27.4$ y $26.7 \%$ al crecimiento total de la región.

La fuerte disminución en la inmigración hacia las regiones Noroeste y Norte, en cambio, propiciaron una tendencia decreciente en el porcentaje que la migración neta aumentó al crecimiento vegetativo; fue mucho más notable en la primera ya que, tras haber agregado $22 \%$ durante el quinquenio $1955-1960$ pasó a agregar sólo $13.5 \%$ dos lustros después. Incluso, después de haber representado la migración neta $18 \%$ del crecimiento total de la Noroeste en el primer quinquenio, pasó a representar sólo $12 \%$ en el tercero.

En la región Centro-Norte (4), en cambio, la fuerte pérdida de población debida a su alta emigración, propició que el crecimiento social negativo redujera $34.8,37.9$ y $39.1 \%$ el crecimiento natural, o en otros términos, que dejara el crecimiento total a $35.2,62.1 \mathrm{y}$ $60.9 \%$ del vegetativo. $\mathrm{Y}$, entre las restantes regiones expulsoras, mientras la migración neta en la Sureste (8) originó cada vez mayores porcentajes reductores del crecimiento natural, en la Occidente (5) y en la Centro (6) fueron cada vez menores. La Noreste (3) fue la única, que tras haber mostrado ganancias por migración durante los dos primeros quinquenios, mostró pérdidas durante el tercero.

\section{CONCLUSIONES}

En este artículo se ha probado la utilidad, mediante una adaptación, del modelo multirregional, para deducir flujos migratorios entre ocho regiones de México para el periodo 1955-1970.

En los resultados de la aplicación, los principales rasgos observados fueron los siguientes:

a) la intensidad de la migración interregional, a nivel nacional, mostró una tendencia ligeramente descendente.

8 Debido a la nula existencia de estudios en la literatura demográfica para México donde se estime el crecimiento natural por regiones, y considerando los problemas de subregistro diferencial en las estadísticas vitales -principal fuente de las estimaciones- es difícil establecer los márgenes de error en las estimaciones que aquí se muestran. 
b) La región Centro-Sur (7) exhibió las mayores tasas de inmigración, sus saldos netos migratorios fueron positivos con respecto a las siete regiones restantes, aumentó cada vez más su ganancia por intercambio con respecto a la Sureste (8), y mantuvo alto su crecimiento social positivo, aunque con tendencia decreciente, con respecto a la Occidente (5) y la Centro (6).

c) La región Centro-Norte (4), la que mayores tasas de emigración y de migración neta negativa exhibió, ambas con tendencias crecientes, mostró cierta reubicación en el destino de sus expulsiones: disminuyendo la intensidad de los flujos hacia las regiones Norte (2) y Noroeste (3) y aumentando la correspondiente hacia la Occidente (5) y la Centro-Sur (7).

d) La fuerte emigración de la Occidente (5) hacia la frontera norte del país ( 1 y 2), exhibió un abrupto descenso en su intensidad con el paso del tiempo, aumentando -posiblemente debido a migraciones de retorno-, también significativamente, su inmigración procedente de las regiones Noroeste (1) y Norte (2).

Las tendencias e intensidad encontradas para los flujos migratorios entre las ocho regiones consideradas, ofrecen una nueva perspectiva para la elaboración de políticas, encaminadas a modificar la migración interna en México y establecer metas deseables de distribución espacial de la población sobre el territorio nacional. Sin embargo, habrá que esperar, para disponer de los resultados del censo de 1980 para todo el país, y deducir de ahí los flujos migratorios interregionales durante la década de los setenta, el gran faltante en las estimaciones mostradas en este artículo.

\section{Bibliografía}

CABrera, Gustavo (1967), "La migración interna en México", 19501960. Aspectos metodológicos y cuantitativos", Demografía y Economia, vol. I (3), núm. 3, pp. 312-367, 1960.

_. "Migración y actividad económica, en México", Cahiers des Ameriques Latines, núm. 12, pp. 1-31, 1975.

_. "Migración interna", Dinámica de la Población de México, Centro de Estudios Económicos y Demográficos, El Colegio de México, México, pp. 85-114, 1981.

CELADE Boletín demográfico, núm. 20, Centro Latinoamericano de Demografía, Santiago de Chile, 1977. 
Ordorica, Manuel, et. al, Migración interna en México, 1960-1970, (Evaluación y Análisis III-5) SIC, DGE, México, 1976.

PARTIDA, Virgilio, "Aplicación del modelo multirregional de población al caso de México", Demografía y Economia, vol. XVI (3), núm. 51, pp. 449-481, México, 1982.

RogERs, Andrei, Matrix analysis of interregional population growth and distribution, University of California Press, Berkeley, 1968.

-Introducción to multirregional mathematical demography, John Wiley, New York, 1975.

SPP, CONAPO y CELADE, México: estimaciones y proyecciones de población, 1950-2000, INEGi, Conapo y Celade, México, 1983.

TABAH León y María Eugenia Cosío, "Medición de la migración interna a través de la información censal: el caso de México", Demografía y Economia, vol. IV (1), núm. 10, pp.43-84, México, 1970.

UNikel, Luis, et al., El desarrollo urbano de México. Diagnóstico e implicaciones futuras, 2a. ed. El Colegio de México, México, 1978.

WAUGH, F. V. y M. E. Abel, "On fraction powers of a matrix", Journal of the American Statistical Association, núm. 62, pp. 1018$1021,1967$. 